\title{
Resultados de intercambio plasmático en síndrome del hombre rígido: experiencia de un caso
}

\author{
Sonia Aznar Barbero, Ramón Bel Cegarra, Josefina Chica Arellano \\ Enfermero/as de la Unidad de Hemodiálisis del Hospital Santa Lucía de Cartagena. Murcia
}

\section{Introducción}

En las últimas décadas la aféresis terapéutica se ha consolidado como procedimiento de depuración sanguínea integrándose en las unidades de nefrología, ya que precisa de un personal entrenado en el manejo del acceso vascular y requiere amplios conocimientos de la circulación extracorpórea y de sus complicaciones ${ }^{1}$.

La plasmaféresis es un procedimiento terapéutico que tiene como principal objetivo eliminar de la sangre aquellos componentes considerados responsables patógenos de una enfermedad y de este modo contribuir a su tratamiento. La técnica consiste en pasar la sangre del paciente a través de un dispositivo extracorpóreo encargado de separar el plasma de los otros componentes de la sangre, eliminando el plasma separado y reemplazándolo en la proporción 1/1 con albúmina al 5\% o plasma fresco congelado².

La aplicación de esta técnica se ha mostrado muy efectiva en el tratamiento de diversas enfermedades neurológicas como el Síndrome de GuillainBarré, Miastenia Gravis y Polineuropatía desmielinizante inflamatoria crónica, con alto grado de recomendación y alta o moderada evidencia. Sin embargo, existen otras enfermedades neurológicas como el Síndrome de Stiff-Person o Síndrome del Hombre Rígido (SHR) en las que la justificación de la aplicación de plasmaféresis se ve reducida a una débil recomendación y baja evidencia (2C) por lo que su uso se ve restringido a aquellos casos en los que las medidas farmacológicas no han obtenido respuesta ${ }^{3}$.

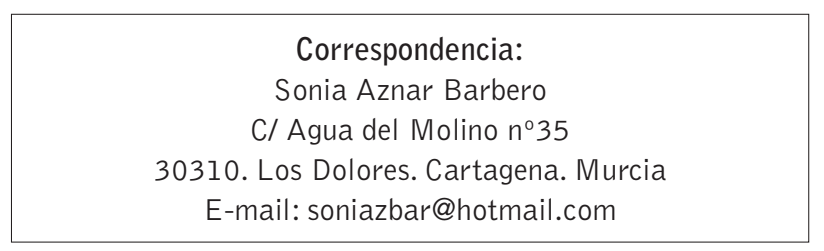

\section{Objetivos}

Presentar el caso clínico de un paciente diagnosticado de SHR (enfermedad poco común) en el que el tratamiento farmacológico convencional no había dado resultados.

\section{Caso Clínico}

Varón de 31 años diagnosticado en 2009 de Síndrome de Stiff-Person atípico o del hombre rígido (SHR): enfermedad invalidante que se caracteriza por una rigidez de la espalda y de las extremidades debida a una continua contracción de los músculos agonistasantagonistas, asociados a espasmos episódicos. En su desarrollo participan anticuerpos dirigidos contra proteínas propias del organismo implicadas en la vía GABAérgica dando lugar a un efecto negativo de la neurotransmisión del ácido gamma aminobutírico (GABA). Su etiopatogenia se ha achacado a cuatro proteínas (dos presinápticas y dos postsinápticas) como posibles autoantígenos ${ }^{4}$.

Con base a estos mecanismos inmunológicos involucrados en la fisiopatogenia de la enfermedad y en la no respuesta a los fármacos inmunomoduladores podría estar justificada la plasmaféresis.

Antecedentes personales: Escoliosis dorso-lumbar y apendicectomizado. No presenta ningún factor de riesgo convencional (no DM, no HTA, no fumador). Trabaja en el mantenimiento y reparación de máquinas expendedoras.

\section{Evolución de la enfermedad}

El paciente acudió a Urgencias en Abril de 2009 refiriendo dolor dorsal intenso que irradiaba de forma proximal y distal por raquis sin desencadenante aparente de 2 meses de evolución tratado por atención primaria con analgesia. En las dos últimas semanas presentó un cuadro de inestabilidad y debilidad en M MII. 
Se le realizó exploración física y control analítico, además de RM de columna dorsal con contraste, $\mathrm{RX}$ de columna cervical y dorsal, RX tórax, ECG y Ecografía de partes blandas en región dorsal diagnosticándose de Dorsalgia muscular y Escoliosis dorsal.

En Junio del mismo año regresó a urgencias por aumento del dolor cervico-dorsal con imposibilidad de mantener la postura erguida y espasmos musculares en MMII y MSI provocando caídas del paciente e interfiriendo en su patrón de sueño. Se le realizaron nuevas pruebas diagnósticas (Analítica de autoinmunidad y serologías, RM columna completa, RM cerebral con gadolinio, RM articulación sacro ilíaca, Ecografía de partes blandas y aparato urinario, TAC de tórax y abdomen, EMG, Interconsultas a Unidad de Raquis, Unidad de Dolor y Unidad de Psiquiatría). Se trasladó al paciente al servicio de reumatología para estudio completo de Ac onconeuronales, AntiGAD y sospecha de Síndrome de Stiff-man sin confirmación, iniciándose tratamiento específico (diacepam, rivotril, toradol, nolotil y adolonta) sin mejoría. Fue dado de alta con ajuste de medicación y recomendación de rehabilitación. Seguimiento por las consultas de reumatología y neurología.

En los dos años siguientes fue tratado por posible Camptocormia con infiltraciones de toxina botulínica cada 4 meses. Presentó Déficit de Vitamina B12. Durante todo este periodo siguieron realizándole pruebas diagnósticas ante la inexistencia de un diagnóstico claro (incluyendo estudios genéticos).

Fue derivado a la Unidad de Neuromuscular del servicio de Neurología del Hospital 12 de 0ctubre (Madrid), Unidad de Trastornos del Movimiento del Hospital Fundación Jiménez Díaz (Madrid) y Unidad de Trastornos del Movimiento del Hospital Clinic (Barcelona) detectándose hiperexcitabilidad muscular continúa sin criterios distónicos ni miopáticos. El paciente comenzó a presentar un cuadro de ansiedad relacionado con el progreso de la enfermedad.

En Junio de 2012 ante el progresivo empeoramiento del paciente se decidió ingreso hospitalario. El paciente ya no era capaz de realizar las ABVD y usaba una silla de ruedas para desplazarse debido a la flexión ventral total del tronco y aumento del dolor.

Fue tratado con varios ciclos de inmunoglobulinas.

En Octubre de 2012 tras ser diagnosticado de Síndrome de Stiff-Person con Camptocormia asociada ingresó por anuria con hipertonía del esfínter vesical siendo dado de alta tras colocación de sonda vesical. Posteriormente se le realizó citostomía percutánea.

El tratamiento farmacológico empleado para esta enfermedad consiste en drogas que incrementan el GABA (ansiolíticos, sedativos y antiepilépticos), drogas antiespásticas, y terapias inmunomoduladoras.

En Abril de 2013 se decidió nuevo ingreso hospitalario para inicio de aféresis terapéutica tras el fracaso de las inmunoglobulinas intravenosas.

El 10 de Abril de 2013 se le implantó catéter provisional yugular derecho y se realizó primera sesión de plasmaféresis con reposición de 3000cc de albúmina al $5 \%$, filtro tipo membrana separador de plasma y anticoagulación con heparina sódica.

Se informó al paciente acerca de los cuidados del acceso vascular resaltando la importancia de mantener la zona seca, evitar tracciones accidentales del catéter y comunicar de forma precoz la aparición de picor y/o dolor en la zona de inserción del catéter, así como de escalofríos, fiebre, sensación de malestar, etc.

La primera semana se realizó aféresis a días alternos, pasando posteriormente a dos sesiones semanales. Dada la buena tolerancia y la excelente respuesta clínica pasó a realizarse procedimiento de aféresis 1 vez por semana (Julio 2013). El paciente recuperó la posición de bipedestación cediendo la contractura de la musculatura abdominal y recuperando la postura erguida, caminando con normalidad. Se le retiró citostomía, obteniendo micción espontánea y normal.

El 3 de Julio de 2013 se le realizó fistula arteriovenosa (FAV) radiocefálica izquierda que comenzó a usarse el 6 de Agosto.

Se informa al paciente de los cuidados de la FAV proporcionándole todos los conocimientos necesarios para detectar las posibles complicaciones.

Al cabo de un mes el paciente acudió a la unidad de diálisis refiriendo dolor en el brazo de la FAV y la desaparición del thrill. Se detecta trombosis de FAV repermeabilizandose con éxito en servicio de cirugía vascular. En Septiembre del mismo año sufrió bacteriemia por S. Epidermidis asociada a catéter temporal yugular que se retiró sin incidencias.

Se ha monitorizado la respuesta a la terapia de aféresis mediante valoración clínica, análisis de Anticuerpos en plasma de anti-GAD67 y anti-GAD65, resonancia magnética del cerebro y estudio neurofisiológico. 
En la actualidad el paciente recibe una sesión mensual de plasmaféresis de forma crónica y ha ido disminuyendo la medicación domiciliaria.

\section{Plan de cuidados:}

Según el modelo de cuidados de Virginia Henderson ${ }^{5}$ establecimos el proceso de atención de enfermería ${ }^{6}$ centrándonos en aquellas necesidades del paciente que no quedaban satisfechas durante la evolución de la enfermedad, tomando como punto de referencia el inicio de la técnica de aféresis:

1. Respirar normalmente: El paciente respiraba con normalidad a lo largo del progreso de la enfermedad. Eupneico. Pruebas de imagen sin hallazgos. Necesidad satisfecha.

2. Comer y beber adecuadamente: EI paciente sufrió una pérdida progresiva de peso conforme evolucionaba la enfermedad.

3. Eliminar por todas las vías corporales: El paciente presentó problemas de estreñimiento derivado del tratamiento farmacológico de control de la enfermedad y un proceso agudo en el sistema urinario.

4. Moverse y mantener posturas adecuadas: Tenía dificultades para deambular debido al dolor intenso causado por las contracturas musculares permanentes y la flexión ventral total del tronco teniendo que usar una silla de ruedas para desplazarse.

5. Dormir y descansar: El dolor continuo consecuencia de los espasmos musculares y de la imposibilidad de mantener la postura erguida provocaron en el paciente alteraciones en su patrón de sueño.

6. Escoger la ropa adecuada, vestirse y desvestirse: A pesar del dolor continuo que presentaba intentaba en todo momento mantener la independencia aunque en ocasiones necesitaba ayuda para satisfacer esta necesidad. Utilizaba ropa cómoda adaptada a sus necesidades.
7. Mantener la temperatura corporal dentro de los límites normales, adecuando la ropa y modificando el ambiente: Presentó un episodio de fiebre durante la bacteriemia derivada del catéter yugular temporal que se trató con antitérmicos, retirada del catéter y antibioticoterapia recuperando su estado basal.

8. Mantener la higiene corporal y la integridad de la piel: Presentaba aspecto limpio y aseado, necesitando ayuda en muchas ocasiones durante el momento del baño.

9. Evitar peligros ambientales y evitar lesiones a otras personas: Se le realizaron numerosas pruebas diagnósticas y tratamientos invasivos.

10. Comunicarse con los demás expresando emociones, necesidades, temores u opiniones: Durante el tratamiento el paciente expresa sus necesidades y sus emociones. Su familia ejerce el rol del cuidador sin mostrar signos de desgaste, apoyándolo en cada momento.

11. Vivir de acuerdo con los propios valores y creencias: necesidad satisfecha a pesar del dolor y la inmovilidad

12. Ocuparse en algo de tal forma que su labor tenga un sentido de realización personal: debido a la evolución incapacitante de la enfermedad el paciente no podía trabajar y manifestaba preocupación por su situación de deterioro progresivo aunque afrontaba de forma positiva el proceso vital.

13. Participar en actividades recreativas.

14. Aprender, descubrir o satisfacer la curiosidad que conduce a un desarrollo normal y a usar los recursos disponibles: mostraba interés por todo lo que le estaba ocurriendo y preguntaba acerca del tratamiento con plasmaféresis. Desde el inicio de la enfermedad ha cumplido estrictamente el régimen terapéutico.

Tabla 1. Plan de cuidados.

\begin{tabular}{|c|c|c|c|}
\hline $\begin{array}{l}\text { Diagnóstico Nanda } \\
\text { (Necesidad 2) }\end{array}$ & Resultados (NOC) & Intervenciones (NIC) & Actividades \\
\hline $\begin{array}{c}00002 \\
\text { Desequilibrio nutricional: } \\
\text { ingesta inferior a las } \\
\text { necesidades } \mathrm{m} / \mathrm{p} \text { peso corporal } \\
\text { inferior en un } 20 \% \text { o más al } \\
\text { peso ideal r/c factores } \\
\text { biológicos y psicológicos }\end{array}$ & $\begin{array}{l}\text { Estado nutricional: } \\
\text { ingestión alimentaria y de } \\
\text { líquidos (1008) } \\
\text { Conducta de aumento de } \\
\text { peso(1626) }\end{array}$ & $\begin{array}{l}\text { Manejo de la nutrición (1100) } \\
\text { Ayuda para ganar peso (1240) }\end{array}$ & $\begin{array}{l}\text { Proporcionar información adecuada acerca } \\
\text { de las necesidades nutricionales y modo de } \\
\text { satisfacerlas } \\
\text { Analizar las posibles causas del bajo peso } \\
\text { corporal }\end{array}$ \\
\hline
\end{tabular}


[ Sonia Aznar Barbero, et al ]

Resultados de intercambio plasmático en síndrome del hombre rígido: experiencia de un caso

\begin{tabular}{|c|c|c|c|}
\hline $\begin{array}{l}\text { Diagnóstico Nanda } \\
\text { (Necesidad 3) }\end{array}$ & Resultados (NOC) & Intervenciones (NIC) & Actividades \\
\hline $\begin{array}{l}0022 \\
\text { Estreñimiento } \mathrm{m} / \mathrm{p} \text { disminución } \\
\text { de la frecuencia r/c hábitos } \\
\text { de defecación irregulares y } \\
\text { actividad física insuficiente. }\end{array}$ & $\begin{array}{c}\text { Eliminación intestinal } \\
(0501)\end{array}$ & $\begin{array}{l}\text { Manejo del estreñimiento/ } \\
\text { impactación (0450) } \\
\text { Manejo intestinal (0430) } \\
\text { Fomento del ejercicio } \\
(0200)\end{array}$ & $\begin{array}{l}\text { Identificar los factores (medicamentos, } \\
\text { reposo en cama y dieta) que pueden causar } \\
\text { estreñimiento. } \\
\text { Instruir al paciente / familia acerca de la } \\
\text { dieta rica en fibras, si procede. } \\
\text { - Ayudar al individuo a desarrollar un } \\
\text { programa de ejercicios de acuerdo a sus } \\
\text { necesidades/capacidades }\end{array}$ \\
\hline $\begin{array}{c}00023 \\
\text { Retención urinaria } \mathrm{m} / \mathrm{p} \\
\text { ausencia de diuresis } \mathrm{r} / \mathrm{c} \\
\text { hipertonía del esfínter vesical }\end{array}$ & $\begin{array}{l}\text { Continencia urinaria } \\
\qquad(0502) \\
\text { Eliminación urinaria } \\
\text { (0503) }\end{array}$ & $\begin{array}{l}\text { Sondaje vesical (0580) } \\
\text { Cuidados de la retención } \\
\text { urinaria (0620) } \\
\text { Manejo de la eliminación } \\
\text { urinaria (0590) }\end{array}$ & $\begin{array}{l}\text { Controlar la ingesta y eliminación. } \\
\text { Mantener una técnica aséptica estricta. } \\
\text { Insertar catéter urinario, si procede. } \\
\text { Remitir al especialista en continencia } \\
\text { urinaria, si procede. } \\
\text { Enseñar al paciente a observar los signos y } \\
\text { síntomas de infección del tracto urinario. }\end{array}$ \\
\hline $\begin{array}{l}\text { Diagnóstico Nanda } \\
\text { (Necesidad 4) }\end{array}$ & Resultados (NOC) & Intervenciones (NIC) & Actividades \\
\hline $\begin{array}{l}00085 \\
\text { Deterioro de la movilidad física } \\
\mathrm{m} / \mathrm{p} \text { movimientos espasmódicos } \\
\mathrm{r} / \mathrm{c} \text { disminución del control } \\
\text { muscular }\end{array}$ & Ambular (0200) & $\begin{array}{l}\text { Terapia de ejercicios: } \\
\text { ambulación (0221) } \\
\text { Fomentar los mecanismos } \\
\text { corporales (0140) } \\
\text { Ayuda al autocuidado } \\
\text { (1800) } \\
\text { Manejo del dolor (1400) } \\
\text { Fomento del ejercicio } \\
(0200)\end{array}$ & $\begin{array}{l}\text { Aplicar / proporcionar un dispositivo de } \\
\text { ayuda (bastón, muletas o silla de ruedas, } \\
\text { etc.) para la deambulación si el paciente } \\
\text { no camina bien. } \\
\text { Controlar la mejoría de la postura / } \\
\text { mecánica corporal del paciente. } \\
\text { Animar al paciente a realizar las } \\
\text { actividades normales de la vida diaria } \\
\text { ajustadas al nivel de capacidad. } \\
\text { Utilizar un enfoque multidisciplinar al } \\
\text { manejo del dolor, cuando corresponda. } \\
\text { Fomentar la manifestación oral de } \\
\text { sentimientos acerca de los ejercicios o la } \\
\text { necesidad de los mismos. } \\
\text { Instruir al paciente acerca del tipo de } \\
\text { ejercicio adecuado para su nivel de } \\
\text { salud, en colaboración con el médico y/o } \\
\text { fisioterapeuta. }\end{array}$ \\
\hline $\begin{array}{l}\text { Diagnóstico Nanda } \\
\text { (Necesidad 5) }\end{array}$ & Resultados (NOC) & Intervenciones (NIC) & Actividades \\
\hline $\begin{array}{c}00095 \\
\text { Insomnio } \mathrm{m} / \mathrm{p} \text { quejas verbales } \\
\text { de dificultad para conciliar el } \\
\text { sueño } \mathrm{r} / \mathrm{c} \text { ansiedad y malestar } \\
\text { físico }\end{array}$ & $\begin{array}{c}\text { Sueño (0004) } \\
\text { Bienestar personal (2002) }\end{array}$ & $\begin{array}{l}\text { Manejo del dolor (1400) } \\
\text { Disminución de la } \\
\text { ansiedad (5820) } \\
\text { Mejorar el sueño (1850) } \\
\text { Aumentar el } \\
\text { afrontamiento (5230) }\end{array}$ & $\begin{array}{l}\text { Fomentar períodos de descanso / sueño } \\
\text { adecuados que faciliten el alivio del dolor. } \\
\text { - Utilizar un enfoque multidisciplinar al } \\
\text { manejo del dolor, cuando corresponda. } \\
\text { - Animar la manifestación de sentimientos, } \\
\text { percepciones y miedos. } \\
\text { - Apoyar el uso de mecanismos de defensa } \\
\text { adecuados. } \\
\text { - Valorar la comprensión del paciente del } \\
\text { proceso de enfermedad }\end{array}$ \\
\hline
\end{tabular}


[ Sonia Aznar Barbero, et al ]

Resultados de intercambio plasmático en síndrome del hombre rígido: experiencia de un caso

\begin{tabular}{|c|c|c|c|}
\hline $\begin{array}{l}\text { Diagnóstico Nanda } \\
\text { (Necesidad 8) }\end{array}$ & Resultados (NOC) & Intervenciones (NIC) & Actividades \\
\hline $\begin{array}{c}00047 \\
\text { Riesgo de deterioro de } \\
\text { la integridad cutánea r/c } \\
\text { inmovilización física y estado } \\
\text { de desequilibrio nutricional } \\
\text { (delgadez) }\end{array}$ & Control del riesgo (1902) & $\begin{array}{l}\text { Enseñanza individual } \\
\qquad(5606)\end{array}$ & $\begin{array}{c}\text { Instruir al paciente y a la familia en un } \\
\text { programa de enseñanza para tratar las } \\
\text { necesidades individuales. }\end{array}$ \\
\hline $\begin{array}{c}00108 \\
\text { Déficit de autocuidado: baño } \\
\text { m/p incapacidad para lavarse } \\
\text { el cuerpo total o parcialmente } \\
\text { r/c debilidad y deterioro } \\
\text { neuromuscular }\end{array}$ & $\begin{array}{c}\text { Autocuidados: baño } \\
\text { (0301) } \\
\text { Adaptación a la } \\
\text { discapacidad física (1308) }\end{array}$ & $\begin{array}{l}\text { Ayuda con los } \\
\text { autocuidados: baño/ } \\
\text { higiene (1801) } \\
\text { Terapia de actividad } \\
\text { (4310) }\end{array}$ & $\begin{array}{c}\text { Proporcionar ayuda hasta que el paciente } \\
\text { sea totalmente capaz de asumir los } \\
\text { autocuidados. } \\
\text { Ayudar al paciente a desarrollar la } \\
\text { automotivación y seguridad }\end{array}$ \\
\hline $\begin{array}{l}\text { Diagnóstico Nanda } \\
\text { (Necesidad 9) }\end{array}$ & Resultados (NOC) & Intervenciones (NIC) & Actividades \\
\hline $\begin{array}{l}00004 \\
\text { Riesgo de infección r/c } \\
\text { enfermedad crónica y } \\
\text { procedimientos invasivos }\end{array}$ & $\begin{array}{l}\text { Conocimiento: control de } \\
\text { la infección (1842) } \\
\text { Control del riesgo: proceso } \\
\text { infeccioso (1924) }\end{array}$ & $\begin{array}{l}\text { Protección contra las } \\
\text { infecciones (6550) } \\
\text { Control de las infecciones } \\
\text { (6540) }\end{array}$ & $\begin{array}{l}\text { Observar el grado de vulnerabilidad del } \\
\text { paciente a las infecciones } \\
\text { Enseñar al paciente y a la familia a evitar } \\
\text { infecciones explicando los cuidados del } \\
\text { acceso vascular necesario para la técnica } \\
\text { de aféresis }\end{array}$ \\
\hline $\begin{array}{c}00146 \\
\text { Ansiedad m/p consciencia } \\
\text { de los síntomas fisiológicos, } \\
\text { sentimientos de incertidumbre } \\
\text { y malestar somático, insomnio } \\
\text { r/c percepción de amenaza } \\
\text { de cambio de rol, estado de } \\
\text { salud, autoconcepto, patrones } \\
\text { de interacción, entorno y } \\
\text { necesidades no satisfechas }\end{array}$ & $\begin{array}{l}\text { Disminución de la } \\
\text { ansiedad (5820) } \\
\text { Aumentar el } \\
\text { afrontamiento (5230) } \\
\text { Enseñanza: proceso de la } \\
\text { enfermedad (5602) } \\
\text { Enseñanza: } \\
\text { procedimiento/tratamiento } \\
\text { (5618) }\end{array}$ & $\begin{array}{l}\text { Ayuda con los } \\
\text { autocuidados: bañol } \\
\text { higiene (1801) } \\
\text { Terapia de actividad } \\
\text { (4310) }\end{array}$ & $\begin{array}{l}\text { Proporcionar información objetiva en } \\
\text { cuanto al diagnóstico, tratamiento y } \\
\text { pronóstico } \\
\text { Animar a la manifestación de sentimientos, } \\
\text { percepciones y miedos } \\
\text { Proporcionar al paciente información sobre } \\
\text { la enfermedad valorando su comprensión } \\
\text { Explicar el propósito del tratamiento } \\
\text { proporcionando toda la información } \\
\text { necesaria e implicando a la familia }\end{array}$ \\
\hline $\begin{array}{l}00155 \\
\text { Riesgo de caídas r/c dificultad } \\
\text { en la marcha y ansiolíticos }\end{array}$ & $\begin{array}{c}\text { Conducta de prevención de } \\
\text { caídas (1909) }\end{array}$ & $\begin{array}{l}\text { Prevención de caídas } \\
\qquad(6490)\end{array}$ & $\begin{array}{l}\text { Instruir al paciente para que pida ayuda al } \\
\text { moverse, si lo precisa }\end{array}$ \\
\hline $\begin{array}{l}\text { Diagnóstico Nanda } \\
\text { (Necesidad 13) }\end{array}$ & Resultados (NOC) & Intervenciones (NIC) & Actividades \\
\hline $\begin{array}{l}00097 \\
\text { Déficit de actividades } \\
\text { recreativas }\end{array}$ & $\begin{array}{l}\text { Implicación social (1503) } \\
\text { Participación en } \\
\text { actividades de ocio (1604) }\end{array}$ & $\begin{array}{l}\text { Terapia de } \\
\text { entretenimiento }(5360)\end{array}$ & $\begin{array}{c}\text { Ayudar al paciente a elegir actividades } \\
\text { recreativas coherentes con sus capacidades } \\
\text { físicas }\end{array}$ \\
\hline
\end{tabular}

\section{Discusión}

En la actualidad la clínica del paciente es el marcador fundamental de la evolución de la respuesta terapéutica siendo el indicador más fidedigno para ajustar la frecuencia de las sesiones de recambio plasmático.

\section{Conclusiones}

La técnica de plasmaféresis se ha mostrado muy eficaz para el tratamiento del Síndrome de Stiff-Person en nuestro paciente aunque la justificación para llevar a cabo el tratamiento tenga una débil justificación y una baja evidencia (2C). El paciente llegó a nuestra unidad tras 4 años de tratamiento farmacológico sin respuesta y tras sufrir un empeoramiento progresivo de su estado físico y emocional. 
Recibido: 20 Agosto 2014

Revisado:25 Agosto 2014

Modificado: 2 Septiembre 2014

Aceptado: 2 Septiembre 2014

\section{Bibliografía}

1. Anaya F. Por qué la aféresis terapéutica debe pertenecer a la especialidad de nefrología. Nefrología 2011; 31 (4): 379-81.

2. Anaya $F$. Manual de aféresis terapéutica basada en la evidencia. Grupo Editorial Nefrología -S.E.N. 2012.
3. Menica $I$, Izaguirre $M \quad F$, Galán $B$, Munerol $C$, Marco A, Gurutxaga $A$ et al. Plasmaféresis en enfermedades neurológicas, a propósito de un caso. Enferm Nefrol 2102; 15 Suppl (1): 102/141.

4. Pérez C, Castañeda Y, Ledesma M. Síndrome de persona rígida y estado de invalidez. Reporte de un caso. Revista Cubana de Salud y Trabajo 2011; 12 (1):66-70.

5. Alba M, Bellido J, Cárdenas V, Muñoz J, López A, Millán $M$ et al. Proceso Enfermero desde el modelo de cuidados de Virginia Henderson y los Lenguajes NNN. Edita: Ilustre Colegio Oficial de Enfermería de Jaén. 2010.

6. Nanda Internacional. Diagnósticos enfermeros: definiciones y clasificación. 2012-2014. Elsevier. 2012. 